\title{
REVIEW
}

\section{Unique Risks, Benefits, and Challenges of Developing Drug-Drug Combination Products in a Pharmaceutical Industrial Setting}

\author{
Nazaneen Pourkavoos
}

To view enhanced content go to www.combitherapy-open.com Received: November 30, 2011 / Published online: March 29, 2012

(c) The Author(s) 2012. This article is published with open access at Springerlink.com

\section{ABSTRACT}

Treatment with a single drug targeting a specific receptor is no longer considered optimal in the treatment and management of complex diseases such as HIV/AIDS, diabetes, and cardiovascular disease. Potential health benefits may arise from the use of affordable, multiple-target, fixedratio drug combinations, which concomitantly reduce multiple risk factors without increasing the risk of adverse effects. Main goals for development of fixed-dose drug combinations may largely be based on the following concepts: treatment of two closely related diseases (e.g., hyperlipidemia and hypertension), patients insufficiently controlled by optimally dosed

N. Pourkavoos $(\bowtie)$

Novo Nordisk A/S, Novo Nordisk Park, DK-2760

Maaloev, Denmark

e-mail: nazp@novonordisk.com

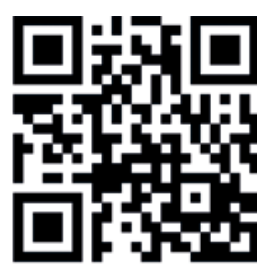

Enhanced content for Combination

Products in Therapy articles is

available on the journal web site:

www.combitherapy-open.com individual monotherapies, and substitution of fixed-dose combination versus free combination. Success in creating and developing the bestin-class commercial combination products requires a multifaceted approach including the following: 1) treatment paradigms in therapeutic area; 2) patient compliance and impact of personalized medicine; 3) shifts in market and cost drivers; 4) preclinical and pharmaceutical development and manufacturing; 5) competitive intellectual property landscape; and 6) clinical development and regulatory filing strategy. Assessment of risks and probability of success of specific drug combinations and drug delivery technologies are needed in order to arrive at the proper recommendation and risk mitigation plans. Personalization of combination therapy is rationalized on the theoretical premise of division of clinical responders from partial or nonresponders to drugs in diseases for which known biomarkers exists. Combination drug therapies with individualized optimization are likely to become a major future focus. A comprehensive review of industrial practice and literature is presented with the goal of developing a best practices roadmap in the feasibility assessment of drug combination therapies. 
Keywords: Antiretroviral; Cardiovascular disease therapies; Diabetes; Dosage form; Drug combinations; Fixed-dose combination; Hepatitis C; Personalized medicine

\section{INTRODUCTION}

Combination products range from drug-drug combinations [1, 2], drug-device combinations such as drug-eluting stents for coronary blockages, and drug-biological product such as monoclonal antibodies combined with a chemotherapy agent for cancer treatment [3-5]. Treatment with a single drug targeting a specific receptor is no longer considered optimal in the treatment and management of complex diseases such as HIV/AIDS, diabetes, and cardiovascular disease. Potential health benefits may arise from the use of affordable, multiple-target, fixed-ratio drug combinations, which concomitantly reduce multiple risk factors without increasing the risk of adverse effects. In the case of an infectious disease such as HIV, the potential benefits of fixed-dose combination (FDC) therapy include the prevention of viral drug resistance by decreasing levels of viral replication and raising the genetic barrier to resistance development.

Increased emphasis on the development and marketing of FDC drugs is an essential component of lifecycle management of both established marketed drugs and new chemical entities for which single-agent and combination studies could advantageously be integrated during early stages of clinical development. Literature analysis of notable FDC candidates in clinical development have largely pointed out the need to fill gaps in product pipelines and to maximize commercial returns of established products owing to patent expiration pressures faced by many pharmaceutical companies.

However, the ultimate success in creating and developing the best-in-class commercial combination products may be achieved by addressing unmet patient needs. This requires a multifaceted approach to the assessment of risks and opportunities in virtually all aspects of the drug development process including the following: 1) treatment paradigms in therapeutic area; 2) patient compliance and impact of personalized medicine; 3) shifts in market and cost drivers; 4) preclinical and pharmaceutical development and manufacturing; 5) competitive intellectual property (IP) landscape; and 6) clinical development and regulatory filing strategy.

In this article, a comprehensive review of industrial practice and supporting literature is presented with the goal of developing a best practices roadmap in the feasibility assessment of drug combination therapies.

\section{METHODS}

The literature search was designed to identify articles and other documents on the development of drug-drug combinations in a pharmaceutical industrial setting as described in the individual sections below. Searches of literature databases and online sources of regulatory information and industrial news, analysis, and perspective were carried out in the context of providing real-life examples from the literature. Multiple information resources and indexes were searched using general and specialized search engines, including Google Scholar and Science.gov. Relevant papers on the topic were categorized, and by examining their bibliographies, additional articles were identified. Applicable journals were reviewed for germane articles on the topic. A commercial reference management software package (Endnote) was used to search Internet databases (PubMed) and manage references. 


\section{TREATMENT PARADIGMS IN THERAPEUTIC AREA}

\section{Justification for Multiple Target \\ Combination Therapy}

Merit for combining various active components in a single-dosage unit is based on therapeutic treatment principles and medical practice. Synergism from combining drugs with different modes of action to treat a disease or diseases may potentially lead to better therapeutic efficacy, improved tolerability by lowering the dose, or by developing new presentations and regimens (e.g., once daily dosing). For example, there is prevalence of overlapping cardiovascular risk factors in a vast proportion of patients with coronary disease and type 2 diabetes. Multimodal combination drugs target a number of risk factors in a single tablet. Diabetes often coexists with dyslipidemia and dyslipidemic patients also suffer from hypertension. Antiretroviral drugs are combined to improve the drug-resistance profile by raising the genetic barrier to resistance development and by preventing development of drug resistance through decreasing levels of viral replication, thereby transforming HIV from a terminal illness to a chronically manageable disease.

The current US Food and Drug Administration (FDA) policy for FDC was established in 1971. The policy states that "two or more drugs may be combined in a single dosage form when each component makes a contribution to the claimed effects and the dosage of each component (amount, frequency, duration) is such that the combination is safe and effective for a significant patient population requiring such concurrent therapy as defined in the labeling for the drug." Essentially these combination modalities fall into three categories concurrent with FDA and European Committee for Proprietary Medicinal
Product guidance's on fixed combination medicinal products $[6,7]$.

\section{First-Tier Category: Improvement of Activity and/or Tolerability}

Drugs in the combination product have different mechanisms of action often with a single aim such that the therapeutic effect is either improved or broadened with a side-effect profile similar to that of respective monotherapies given at the same or higher dose. For example a recent study found that the triple-drug combination of olmesartan/amlodipine/hydrochlorothiazide for the treatment of hypertension was very effective, safe, and well tolerated by patients [8]. Other recent clinical reviews of new drug medications include effectiveness and safety of two drug combinations for type 2 diabetes [9] and for dyslipidemia and obesity treatment in metabolic syndrome [10]. Alternatively, tolerance of a lowdose combination of drugs is improved with similar therapeutic effectiveness over respective higher dose monotherapies [11].

Examples of well-established FDC that are approved or in late-stage development, as well as new single agent monotherapies, and FDC in current pipeline for prominent therapeutic areas are provided in Tables 1-4. The information includes the potency of each therapeutic agent, mode of action, current phase, trade name, and companies for therapies in cardiovascular, HIV/AIDS, hepatitis diseases, and diabetes (Tables 1-4, respectively).

\section{Second-Tier Category: Improvement in Symptomatic and Pharmacokinetic Profile} These combinations may contain at least one component not previously approved for an indication. The intensity and duration of action of monotherapy is increased by combining with a second component that reduces the metabolic inactivation or elimination of the 


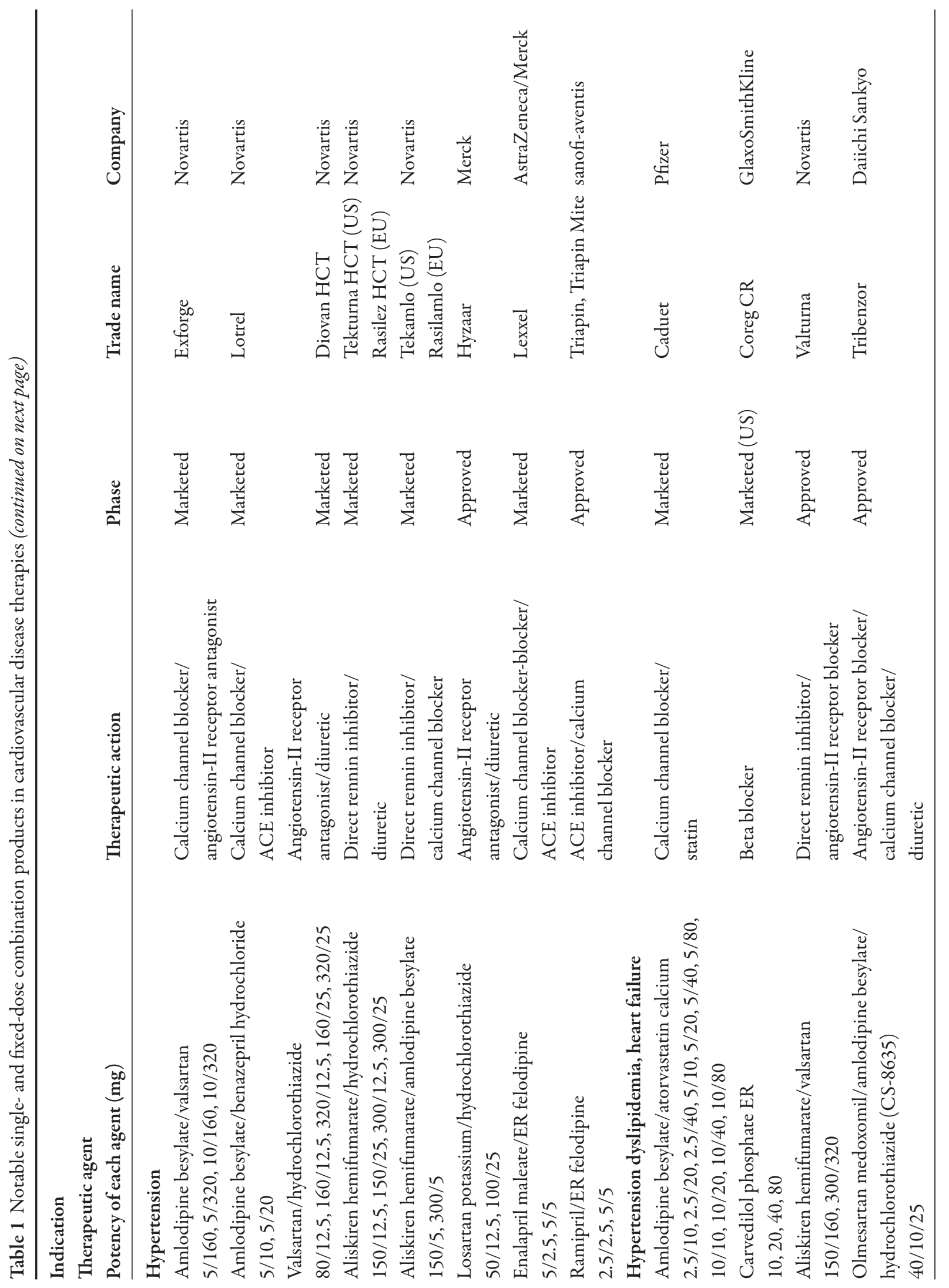




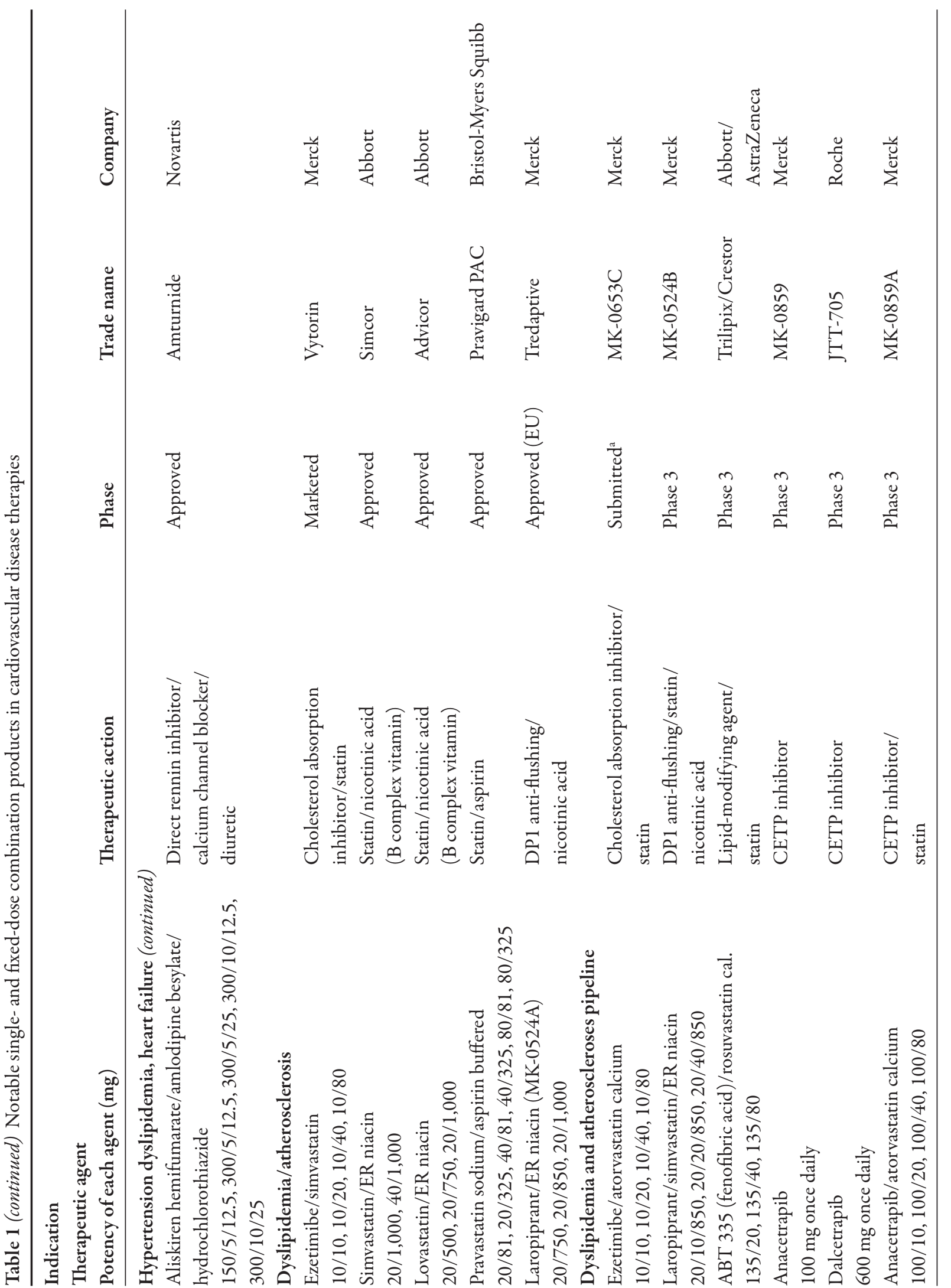




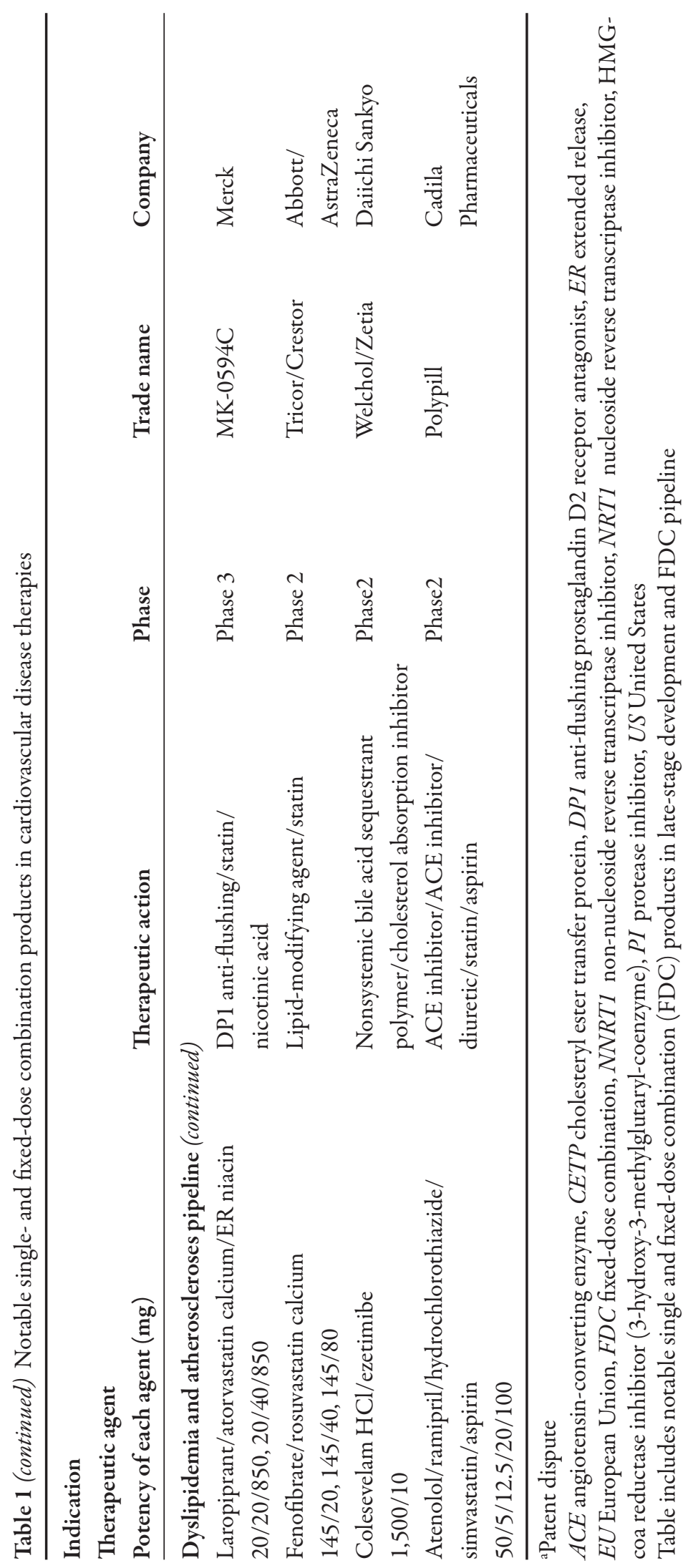



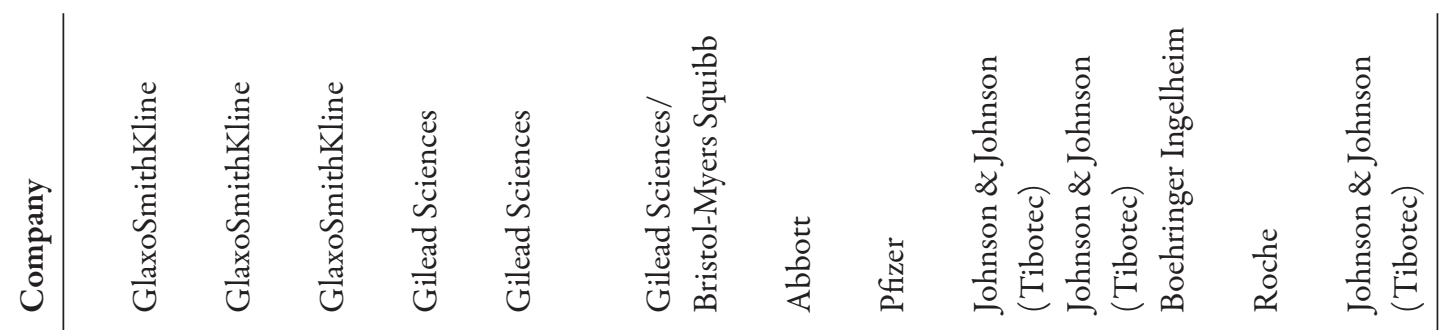



䓪

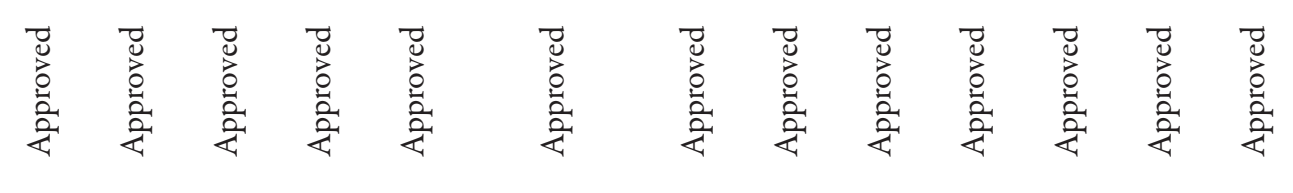

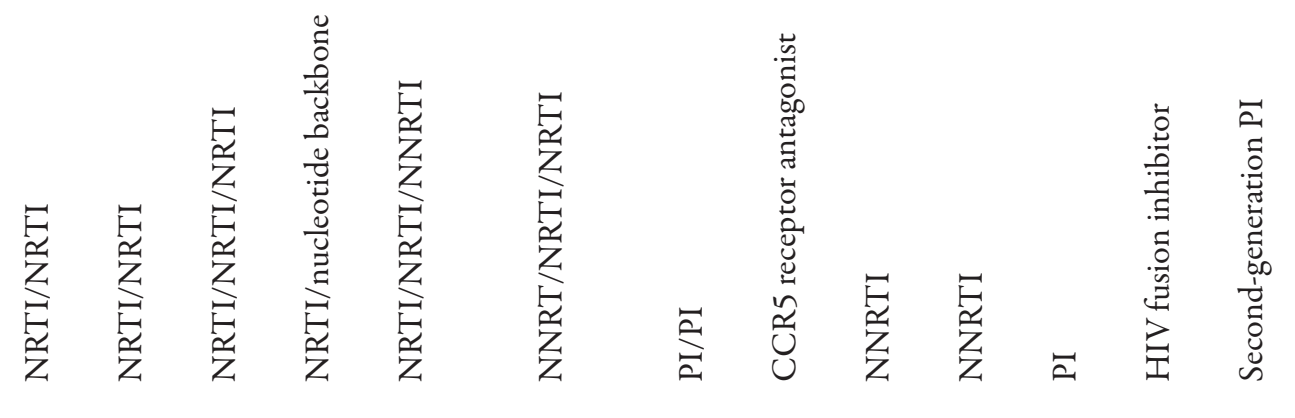




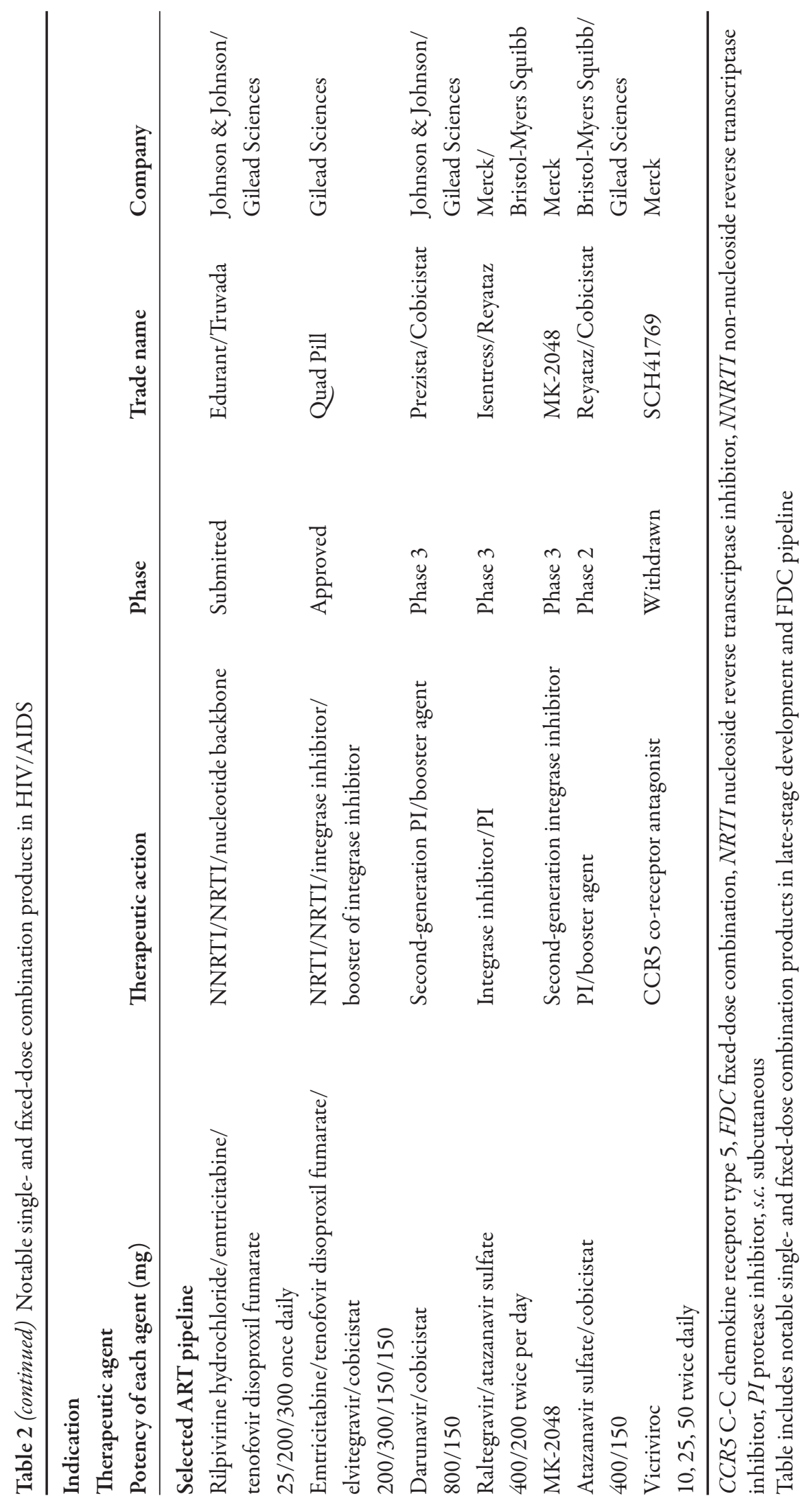




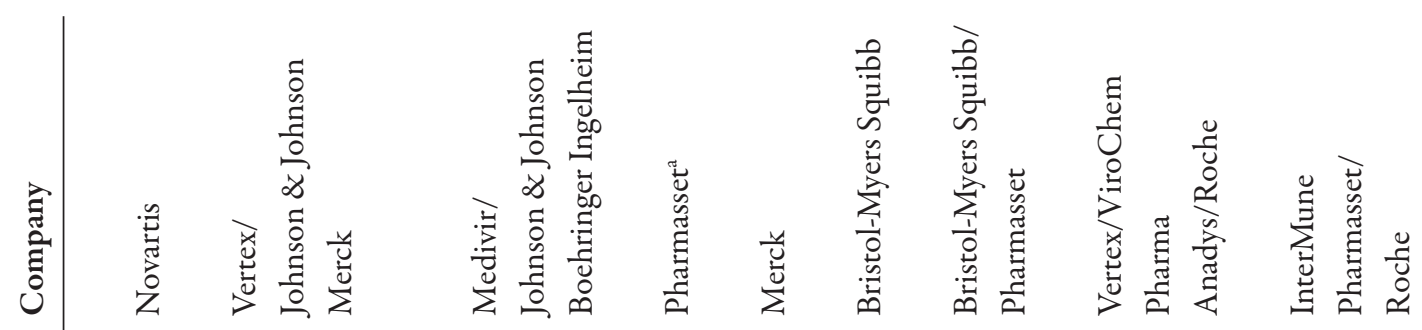

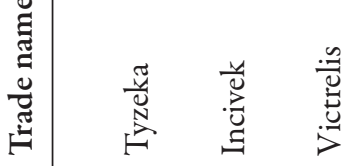

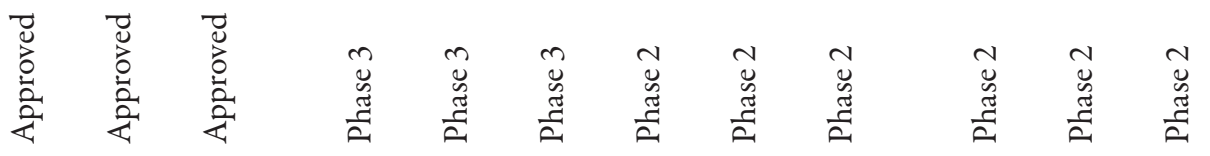

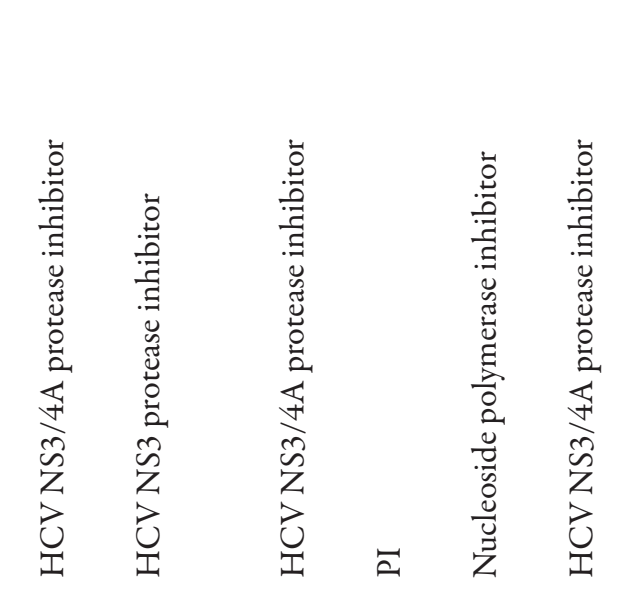

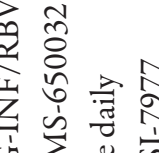




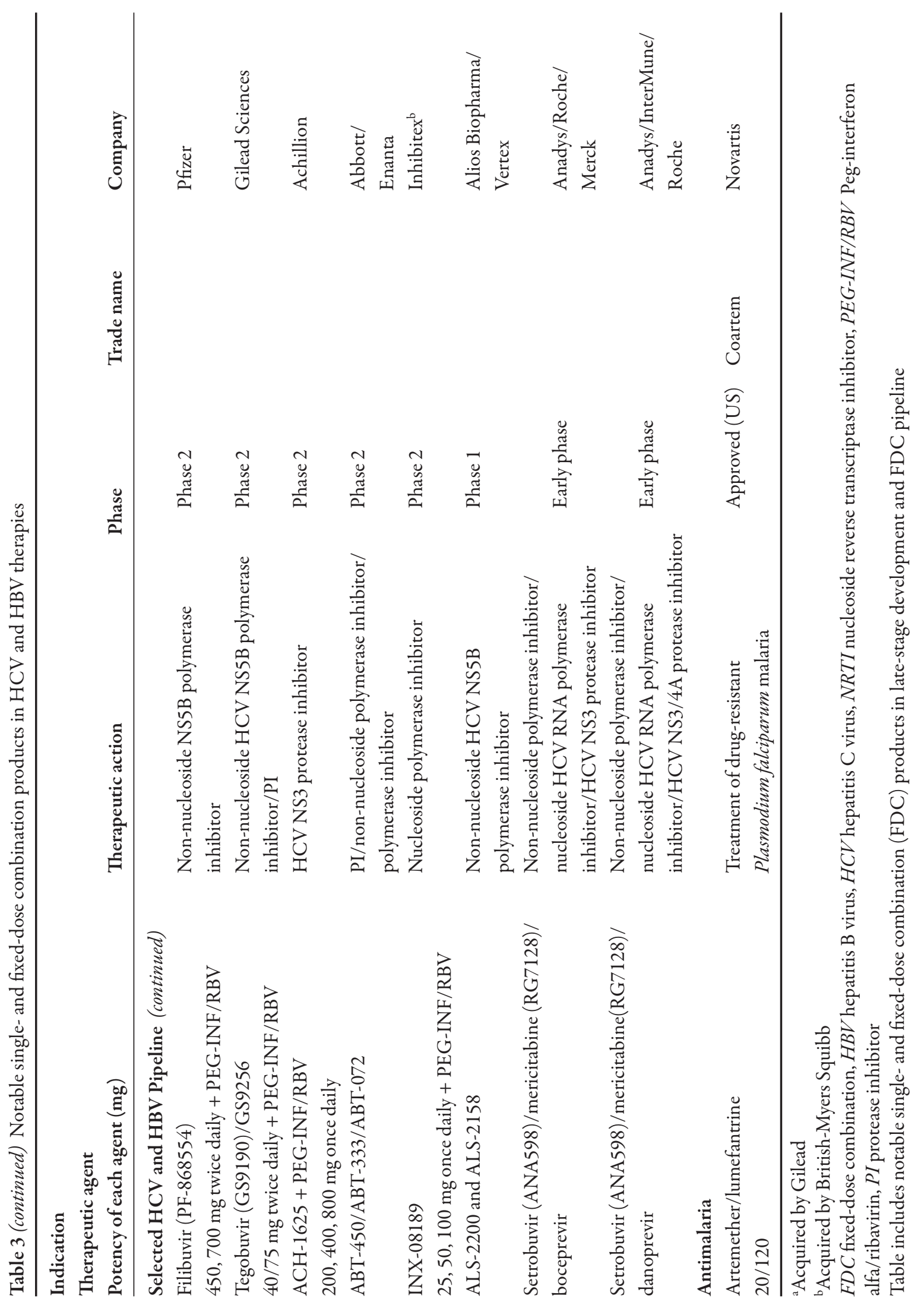




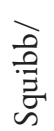

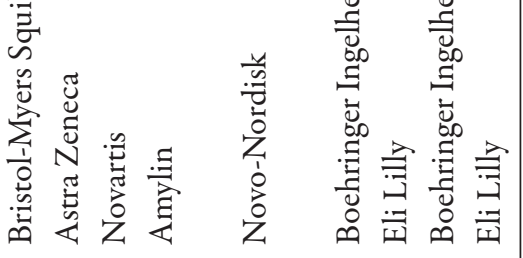

: 咅焉

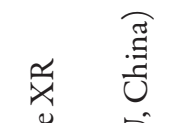

U

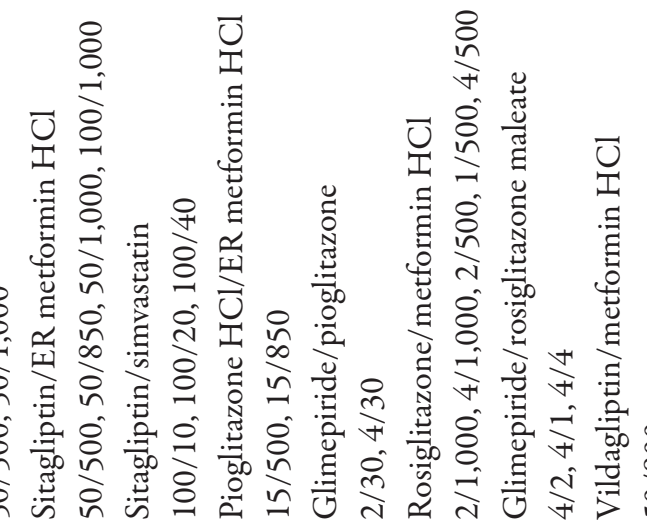

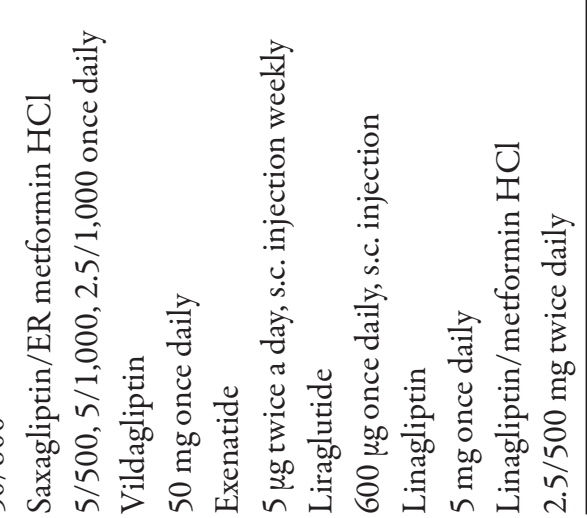




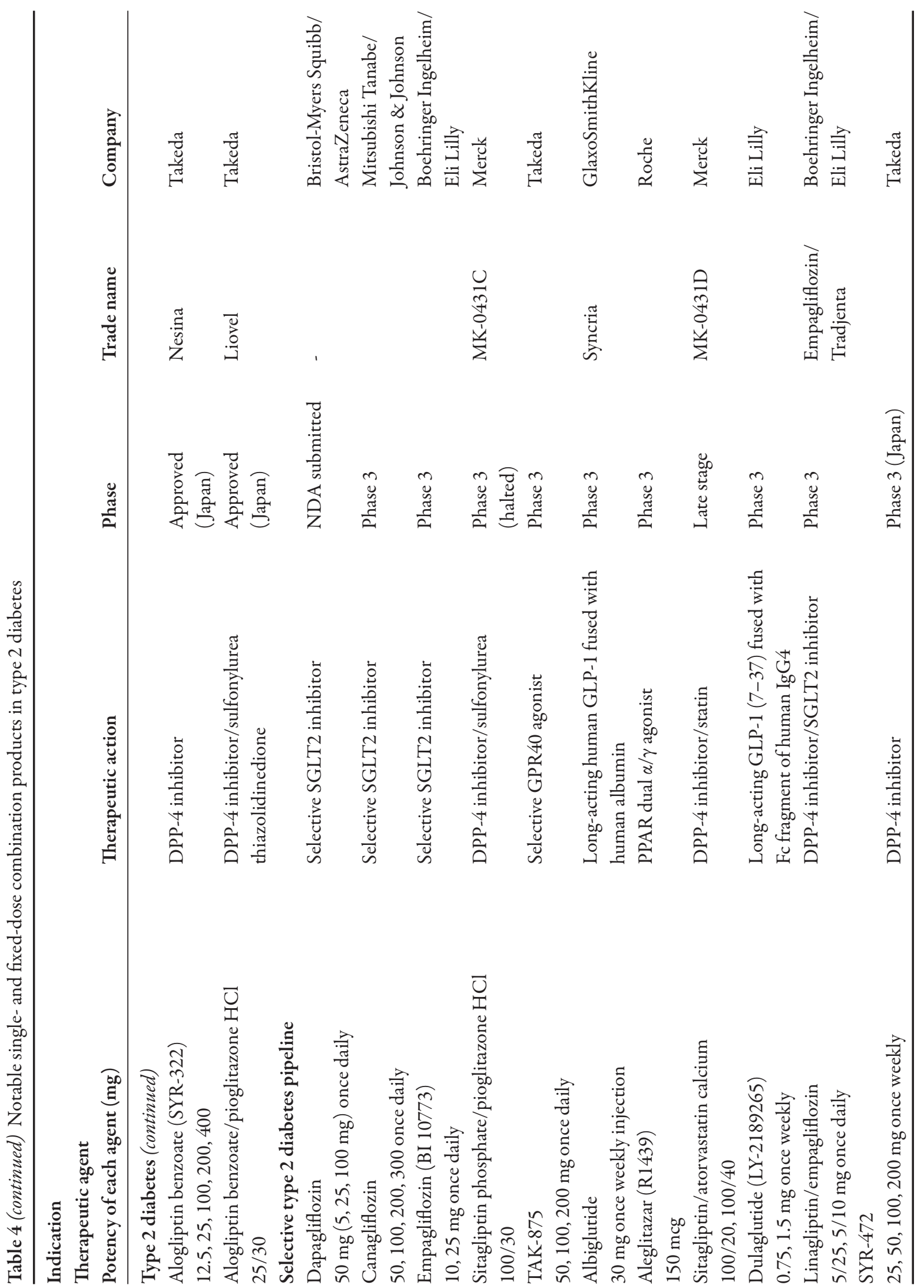




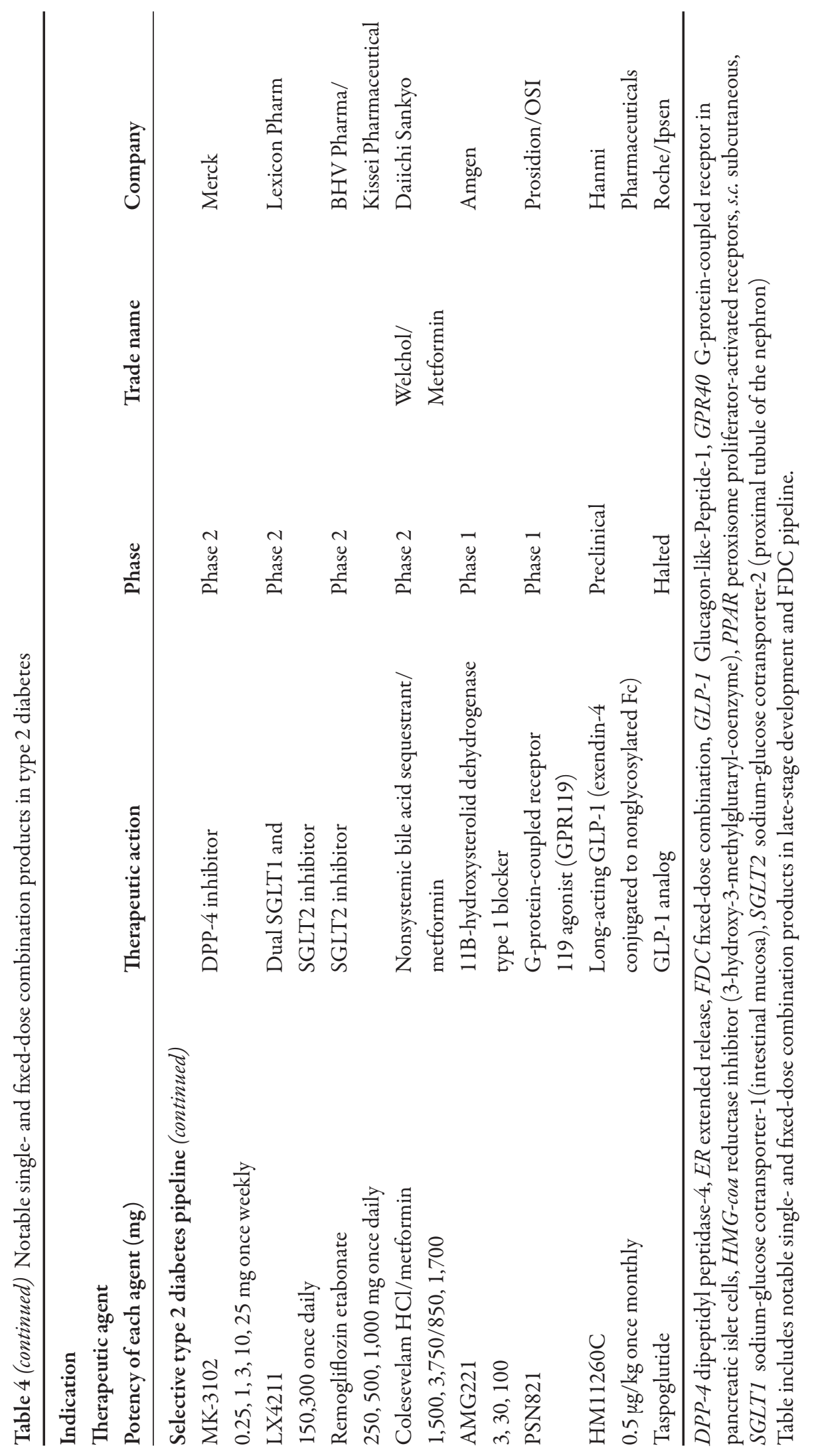


other agent or agents, such as the addition of the booster agents, ritonavir and cobicistat (GS 9350), to enhance the antiretroviral (ARV) effect of protease and integrase inhibitors [12]. Alternatively, a component in the FDC acts by diminishing the dose-related side effects of the other agent such as the addition of laropiprant to a FDC of niacin and statin to counteract the skin-flushing effect of high-dose niacin.

\section{Third-Tier Category: Simplification of Dosage Regimen}

In general, regulatory guidelines have demerited FDC products that promote convenience claims without providing additional health benefits. However, improving compliance and adherence to therapy brought about by reducing the pill burden or simplifying the dosing regimen, such as once daily, may be sufficient proof to provide regulatory flexibility. The issue of adherence is particularly important in the areas of infectious diseases and diabetes where inadequate or inconsistent adherence to multiple therapy and dosing regimen may lead to the development of viral resistance and inadequate glycemic control, respectively $[13,14]$.

\section{Inherent Risks of Combination Products}

\section{Selection of the Most Appropriate Therapeutic Agents}

Arguably the foremost risk factor is in the selection of the most appropriate therapeutic agents intended for development as a combination product. In other words, does the proposed combination of drugs and doses represent the most clinically appropriate choice of drugs based on available efficacy, safety, and cost data? If this first condition is not satisfactorily vetted, it is possible that a less optimal FDC is chosen because of its availability and the perceived improved adherence rather than its demonstrated clinical benefits. A less optimal FDC may also occur when pharmaceutical companies develop combination drugs exclusively from their own portfolio whereas more viable alternative drugs from other companies are overlooked. Recent examples are montelukast/loratadine FDC for allergic rhinitis, and niacin/laropiprant for hypercholesteremia.

\section{Multiple Drug-Drug Interactions}

The administration of FDC raises the risk of multiple drug-drug interactions and unexpected adverse events. Pharmacokinetic and pharmacodynamic data are needed to assess adverse effects of drug-drug interactions particularly in high-risk subgroups, such as the elderly and patients with diabetes and/or renal disease [15]. Only for established drugs that are often prescribed concomitantly, welldocumented bibliographic data may provide a rationale to minimize the number of new studies needed to establish safety and efficacy of the proposed combination.

\section{Loss of Therapeutic Flexibility}

Another risk is loss of therapeutic flexibility for titration. Combining agents with different therapeutic targets, such as hyperlipidemia and hypertension, require dose titration of each drug component. Developing too many dose combinations could defeat the object of developing a simplified combination therapy. For example, Pfizer (NY, USA) developed 11 different dosing combinations of Caduet ${ }^{\circledR}$ (amlodipine besylate/atorvastatin calcium) to accommodate this need [16].

\section{Life Cycle Management (LCM) Development Strategy}

If LCM strategies are left late in the development cycle until close to patent expiration, there is a business risk that the developed FDC may quickly 
be superseded by other drug combinations over time in a competitive landscape. For example, ARV regimens of two drug combinations were superseded by three drug combinations. "Quad pill" was developed by Gilead Sciences (Foster City, CA, USA) that combines four distinct ARV components in a single dosage (Table 2). Also, a potential novel combination with new chemical entities requires early integration of complex preclinical and clinical development programs.

\section{Patient Acceptance Criteria}

For oral medications, there is a risk that the combination tablet may be too big for some patients to swallow, particularly if more than one large unit is to be taken together at once. Such risks can be mitigated to some extent by dosage formulation design and manufacturing technology to minimize physical size and dimensions and by conducting preliminary market research based on sight samples of the FDC and its comparison to other large-size medications on the market.

\section{Patient Compliance and Impact of Personalized Medicine}

From the patient's perspective, polypharmacy and the complexity of the medication regimen are important factors for noncompliance [17]. The incentive for simplification of therapy is to improve compliance and adherence to medication in order to improve health outcomes [18]. The relationship between adherence to ARV combination regimen and development of drug-resistant viral mutations and replication was reviewed by Bangsberg et al. [19].

The National Council on Patient Information and Education has estimated that because of poor adherence between $30-50 \%$ of prescriptions fail to produce the desired therapeutic results in patients with chronic medical conditions [20]. Several studies in compliance literature have provided data to support this theory [21, 22]. Bangalore et al. [23] conducted a review of randomized, controlled trials and a retrospective analysis of medication compliance for nine studies that compared FDC with free-drug regimens of the same medication. The chronic conditions were hypertension in four studies, diabetes in two studies, tuberculosis in two studies, and HIV infection in one study. A total of 11,925 patients on FDC were compared against 8,317 patients on monotherapy regimens. The meta-analysis showed that FDC resulted in a 24-26\% greater patient compliance across all groups.

\section{Impact of Personal Medicine}

An ultimate paradigm is to personalize treatment to individual circumstances. Each patient has unique characteristics, such as weight, age, pharmacogenetics, and co-existing illnesses, and genetic polymorphism affecting drug metabolism, transport, and effect (both efficacious and adverse). This may argue for close monitoring, and optimization of dose and frequency of individual drugs as opposed to administration of a FDC tablet. However, personalization of combination therapy is rationalized on theoretical premise of division of clinical responders from partial or nonresponders to drugs in diseases for which known biomarkers exists $[24,25]$.

Pharmacogenomics seeks to identify and validate molecular diagnostic assays/tools for defining pathogenesis and stratifying patient population into treatment responders and nonresponders. Clinical diseases in which personalized medicine is taking an increasing prominent role includes cancer treatment, diabetes, cardiology, and neurology [26]. 
Although the impact of personalized medicine on practical strategies used to design combination products remains to be seen, initial emphasis may focus on anticancer drugs and the development of concomitant companion diagnostics. In this respect, FDA draft guidance on in-vitro companion diagnostic devices points to several key features [27]. First, it defines in-vitro companion diagnostics (IVDs) as devices that provide essential information for the safe and effective use of corresponding therapeutic products, by identifying individuals most likely to benefit or suffer adverse reactions from treatment. Second, it states that FDA will review targeted drugs for approval only in the context of their corresponding IVDs. Third, it aims to clarify drug labeling by identifying what types of FDA-approved IVDs are appropriate for use in selecting patients and their monitoring.

Recent such examples include: 1) approval of vemurafenib and multiple polymerase chain reaction (PCR)-based diagnostic for BRAF V600 E gene for advanced melanoma harboring the mutation, and 2) crizotinib for non-small cell lung cancer in patients with tumors containing anaplastic lymphoma kinase (ALK) gene with a fluorescent in situ hybridization (FISH) test for detecting rearrangements of the ALK gene [28].

The realization of these expectations requires medications with a biomarker test, and largescale patient data on associations between genotypes and drug-response phenotypes from clinical trials [29-31]. Researchers have called for forming knowledge management infrastructure systems devoted to archiving and analyzing pharmacogenetics data from many thousands of individuals toward tools for personalized medicine.

A bioinformatics research pilot study in Huntington's disease was established at GE Global Research Center to develop predictive models that combined medical informatics; i.e., methods to identify statistical correlations within clinical data with bioinformatics, which provides knowledge of polymorphism in genes that encode proteins that are the target of the proposed drug [32]. The Marshfield Clinic Personalized Medicine Research Project (PMRP) represents one of the largest populationbased DNA biobanks in the United States. Wilke et al. [33], as part of an effort to begin phenotyping common diseases such as diabetes within the PMRP database, constructed a diabetes case-finding risk algorithm that utilized diabetes diagnostic codes in combination with clinical laboratory data and medication history. This algorithm yielded an estimated prevalence of $24.2 \%$ for diabetes mellitus in adult subjects 50 years or older. The implication is to identify individuals for targeted drug therapy either from less effective therapies or by identifying less symptomatic individuals who might benefit from prophylactic treatment (no antidiabetic drugs are presently licensed for use in prediabetic individuals).

As previously discussed, drug and biomarker diagnostic combinations (also termed theranostics) have been categorized on the basis of their use for clinical response/ efficacy by differentiating potential responders from nonresponders, drug susceptibility, and resistance, and dose selection or adjustment to optimize drug efficacy and safety. The FDA calls for two types of testing: 1) required tests before prescribing specific drugs that are useful in identifying potential responders in whom drugs are shown to have an enhanced efficacy, and 2) recommended tests that assist with dose selection/adjustment to primarily prevent a drug's severe adverse effects [34]. Further examples of the tests that are in current use are provided below:

1) Monogram Bioscience's Trofile Assay for evaluating a patient's antibody response to 
envelope proteins of a virus was developed as a means to identify responders to maraviroc a C-C chemokine receptor type 5 (CCR5) coreceptor antagonist compound for treatment of HIV $[35,36]$.

2) Determination of individual genetic characteristics of the tumor allows for possible tailoring of cancer treatment. Gene expression profiling studies have provided a molecular classification of breast cancer into clinically relevant subtypes for predicting the benefits of chemotherapy in newly diagnosed breast cancer patients. The commercially available Genomic Health's Oncotype DX 21 gene expression assay was shown to predict endocrine therapy response and recurrence out to 10 years in patients with estrogen receptor-positive, and lymph node-negative breast cancer. The clinical trial assigning individualized options for treatment was a large, multicenter, randomized trial (TAILORx) that used this genomic test [37-40]. More recently this was expanded to the use of a 70-gene expression pattern predictor as a prognostic factor in breast cancer recurrence and patient stratification to low and high-risk groups [41].

3) A strong association was found between genetic polymorphism in the recently identified vitamin $\mathrm{K}$ epoxide reductase complex 1 (VKORC1) gene and interindividual variability in the adverse reactions to anticoagulant effect of warfarin. Genotyping test has made it possible to identify the variants in gene encoding (VKORC1 haplotypes) responsible, in part, for coumarin dose-adjustment requirements [42].

4) Niemann-Pick C1-like 1 (NPC1L1) gene encodes a intestinal sterol transporter, which is the likely target for ezetimibe, a cholesterol uptake inhibitor. DNA sequence variation in the NPC1L1 gene (several single nucleotide polymorphisms [SNP]) was associated with the improved low-density lipoprotein (LDL) lowering clinical response to treatment with ezetimibe. The association between changes in plasma sitosterol levels (a direct marker of cholesterol absorption) and SNP haplotypes were carried out in Caucasians in the ezetimibe add-on to statin for effectiveness (EASE) trial and vytorin versus atorvastatin (VYVA) trial, and in the two trials combined $[43,44]$.

5) DNA variation in organic cation transporter 1 (OCT1) absorption, distribution, metabolism, and elimination (ADME) gene affects response to metformin, which is widely used as the first-line drug in treatment of type 2 diabetes mellitus [45].

6) Other examples of genetic marker tests that are used to identify ethnic and racial differences in clinical response include the following cases: systemic administration of trastuzumab an anti-HER2 antibody in patients with the HER-2/neu (encodes a epidermal growth factor receptor protein) overexpressing breast cancer tumors [46]; a prognostic biomarker test used to detect polymorphism amongst patients with genotype 1 hepatitis $C$ infection (a single allele change in a tiny segment of DNA code near the Interleukin-28B gene) guides treatment decisions to standard hepatitis $\mathrm{C}$ regimen (i.e., a combination of pegintron and the antiviral ribavirin). An application to use a genetic marker test to screen patients that might develop liver damage from taking lumiracoxib was recently resubmitted to FDA by Novartis AG.

Despite numerous scientific and implementation challenges for translating the concept of personalized medicine into clinical practice, progress is being made in providing structure for achieving consensus on public policy positions among stakeholders $[47,48]$. 


\section{MARKET SHIFTS AND DRIVERS}

It is well recognized that the overall costs for development of new chemical entities have continued to rise. Estimates of actual costs vary but that on average it represents $\$ 750$ million to $\$ 1.1$ billion research and development expenditure by the pharmaceutical industry. At the same time, loss of molecules and revenue due to patent expiration is outpacing the introduction of new ones to the market. Pharmaceutical companies are seeking different ways of revenue generation to offset this imbalance. LCM is seen as an essential strategy of drug franchise development, product revitalization, and maintenance or increase in market share. Generally, new combinations of established drugs carry low risk due to lower development costs (i.e., \$10-50 million) and less burdensome regulatory path to approval since in many cases safety and efficacy of each individual marketed drug has been established. These combinations, if truly shown to satisfy an unmet need, create and extend value because of speed to market and generation of new patents that may protect from generic competition.

Cost-effectiveness of FDC in the outpatient setting is seen in terms of avoidance of medication errors (missed or omitted doses), improving adherence, and efficacy. Newman et al. applied decision modeling to analyze the cost-effectiveness of four FDCs for primary cardiovascular disease prevention in men which found FDCs to be cost-effective for men older than 55 years [49]. Securing favorable pharmacoeconomic coverage for FDC can be very challenging in today's drug benefit and coverage trends but this may depend on therapeutic area, availability of generic versions of single agents, pricing, and other factors.

\section{KEY PHARMACEUTICAL FORMULATION AND MANUFACTURING CHALLENGES}

Formulation development and manufacturing of multi-active component dosage forms present many unique challenges. These challenges may arise due to following scenarios: 1) physicochemical incompatibility of the active pharmaceutical ingredients (APIs) with each other and with the common excipients used in the FDC formulation; 2) changes in the rate and extent of in-vitro dissolution and in-vivo bioavailability of APIs from FDC as compared to that observed for the single component formulations of individual APIs; 3) undesirable mechanical powder flow and compression characteristics of the multiactive granulation/powder blend; and 4) increase in dosage bulk volume due to additional drug loading particularly for high-dose combinations.

For these reasons, a conventional tablet or capsule dosage form may rarely suffice to meet the various stability, bioequivalency, and commercial manufacturing requirements. Combination of APIs classified as Biopharmaceutics Classification System (BCS) Class II and IV are particularly challenging and require specialized solubility enhanced formulations and amorphous conversion processing technologies that are capable of providing stable, bioavailable, and scalable formulation to accommodate the range of drug loading and potencies of each API. There could be also requirements for individual modification of drug release and pharmacokinetic profiles such that a combination of both immediate and extended drug release formulations may be needed for the combination dosage form.

If an API is initially formulated as a liquid or semi-solid encapsulated composition, its reformulation into a biocomparable solid state formulation could be a prerequisite in order to 
make the combination with other drugs viable. A comparative in-vivo bioavailability study of liquid versus solid reformulation having a similar food effect can be used to determine if a modest upward potency adjustment can be supported by prior preclinical toxicological safety data to achieve a bioequivalent pharmacokinetic profile for the reformulated product. For BCS Class II and IV compounds, this may involve use of complex solid solubilization co-processing techniques such nanomilling, amorphous API solubilized polymeric dispersions, and surfactant co-granulation technologies.

Multilayer tablet technology is particularly useful for formulating FDC. It allows for compression of separate drug layers, thus minimizing physicochemical incompatibility and stability problems that may arise from the intimate contact of individual drug compositions. Atripla $^{\circledR}$ (Gilead Sciences, Foster City, CA, USA) comprises of a dry granulation layer of tenofovir disoproxil fumarate and emtricitabine and a wet surfactant co-granulation layer of efavirenz [50]. Bilayer tablet technology also provides flexibility in combining formulations with sustained-release and immediate-release layers in the same dosage unit. This was utilized in developing combinations of extended release niacin (controlled-release layer) and immediaterelease layer of laropiprant with simvastatin or atorvastatin (Tredaptive in European Union [EU]). It is noted that physical and mechanical aspects of bilayer tablet compression is more complex than conventional single layer tablet compression. Challenges encountered are due to weight control of the thinner drug layer compression fill weight, and interfacial delamination. The latter may occur as a result of inadequate interfacial bonding strength immediately following ejection or upon further processing stress and attrition such as in pan film coating operation. Delamination may also occur due to differential expansion of the layers upon exposure to elevated humidity conditions upon storage and stability testing.

A strategy to reduce dosage size and enhance swallow ability is to apply precision film coating to deposit API as a polymer film layer (rather than a separate granulation layer) over a separate drug containing tablet core [51]. The drug core could be an extended release matrix core, a conventional tablet core, or a bilayer tablet. These formulation designs provide flexibility for various scenarios of combining multiple APIs, dosing combinations and drug release rates.

FDC development strategies for new chemical entities (NCEs) are increasingly being incorporated early during the compound development program, so that formulation design activities and manufacturing process development for both monotherapy and combination are performed in parallel. This strategy aims to avoid formulation switching and bioavailability issues from occurring late in the development program and commercial stages. However, in many cases FDC development takes place after initial approval of monotherapy is obtained. Therefore, existing knowledge of APIs preformulation and dosage form formulation and stability mechanisms can be heavily leveraged in experimental design to streamline and fast track FDC development and regulatory filing as much as possible.

A further strategy to minimize formulation development particularly when drug potencies are low is to incorporate them in a multiphase capsule technology, which involves co-encapsulation of existing tablet/powder blend/multiparticulates components in potentially sealed compartments. For example, Lotrel $^{\circledR}$ (Novartis, East Hanover, NJ, USA) is marketed as a hard gelatin co-encapsulation of benazepril hydrochloride film-coated tablet and amlodipine besylate granulation powder blend. 


\section{FDC Formulation, Manufacturing, and} Regulatory Development Roadmap

There are common strategies and approaches that could be taken to successfully develop and market FDC products. The initial project scope and gap analysis activities could be initiated by a review of internal therapeutic area portfolio and by identifying unmet patient needs, new data or indication arising from clinical research and/or clinical practice setting as well as market and brand opportunities. Following gap analysis, the next steps are idea generation, prioritization, and a preliminary review of related API and formulation competitive IP landscape that may depend on combining approved drugs or NCEs. Assessment of risks and probability of success of specific drug combinations and drug delivery technologies are needed in order to arrive at proper recommendation and risk-mitigation plan.

These multidisciplinary plans are used to obtain initial organizational go/no-go decision to allow for allocation of resource funding pending more detailed technical and manufacturing reviews. This could be achieved by conducting a paper feasibility exercise focusing on technical API, formulation and process design aspects as well as regulatory and manufacturing challenges and opportunities for resolution. The paper feasibility assessment may contain several sections from information collected or generated during the prior project scoping and idea generation stages:1) target product profile; 2) API sourcing; 3) preclinical safety pharmacology considerations; 4) FDC formulation and process design; 5) IP issues; and 6) regulatory filing strategy.

\section{Target Product Profile}

Target product profile comprises the first step in broadly defining objectives and requirements for meeting product target profile in terms of goals set for disease indication, clinical efficacy, safety, potency, route and frequency of administration, bioequivalencey criteria, and shelf-life stability. These objectives are aligned with organization of sections in the product's label.

Target product quality profile (TPQP) aims to predefine chemistry, manufacturing and controls (CMC) requirements and performance criteria for product critical quality attributes (CQA) that focuses on guiding formulation, and manufacturing process development efforts. TPQP can be inclusive of predefining drug substance physical form, its biopharmaceutical properties, as well as dosage form characteristics, such as assay, content uniformity limits, compression properties, microbial limits, and impurity/degradant projected specification limits. In addition, dosage form performance criteria such as in vitro-dissolution profile, in-vivo bioavailability profile, stability, and package requirements are included [52]. These critical quality product attributes are refined and revised based on new data that are generated during the later experimental and development stages.

\section{API Sourcing}

In some situations a FDC product may involve combining an externally sourced API with therapeutic agent/s from innovator's own internal development portfolio (whether marketed or an NCE). Externally sourced API often presents challenges in terms of its procurement. Such issues and risk-mitigation plans can often be considered in advance during the feasibility assessment stage and may include the following areas: 1) API vendor selection, vendor suitability, and status of prior quality assurance assessment if any, and procurement lead times and cost factors; 2) availability of drug master files for 
investigational new drug regulatory filing in US, as well as early identification of IP factors that may require sourcing from overseas API vendors; 3) availability of Good Manufacturing Practice (GMP) quality and non-GMP material meeting standard pharmacopeia specifications as needed (US Pharmacopeia [USP], EU, Japanese Pharmacopeia [JP]) for conducting initial feasibility and stability studies; 4) applicability of drug importation requirements, such as securing in advance, investigational new drug application approval from regulatory authority for importation of GMP-quality material for human studies.

\section{FDC Formulation and Process Design}

Design of multi-active combination products requires knowledge of each individual API physicochemical and biopharmaceutical properties as well as sources and mechanism/s of chemical degradation and physical instability. API physicochemical profiling data are used to predict associated developability risks, and formulation complexity factors. For BCS Class II and IV compounds, initial selection of preferred solubilization enhancing technologies and inclusion of functional excipients depend on API physico-chemical and gastrointestinal (GI) physiological factors [53]. Traditional high-throughput robotic salt, and polymorph screens are supplemented by solubility screens of lead forms in various $\mathrm{pH}$ media, surfactant and vehicles that are intended to inform on solubility and dissolution of API (and its salt) forms as well as API-excipient blends under gastrointestinal relevant conditions [54-56]. The outcome is lead formulations that can achieve dissolution and supersaturation.

Selection of appropriate API phase, salt form and physical state together with API solubility data in physiologically relevant $\mathrm{pH}$ media (encountered in fasted stomach and proximal intestine), as well as estimated clinical dose solubility ratio, and intrinsic permeability can be used to forecast whether the oral absorption process is predominately limited by apparent solubility or by dissolution rate. Such predictions are increasingly made using physiologicallybased drug absorption modeling (PBPK), which represents the compartmental absorption and transit model (ACAT) implemented in the commercially available software such as GastroPlus, (Simulations Plus, Lancaster, CA, USA), and PK-Sim (Bayer Technology Services GmbH, Leverkusen, Germany) $[57,58]$.

Computational absorption simulations particularly when coupled with input parameter sensitivity analysis that for example simulates exposure for a range of doses and particle sizes may point to viable formulation options that are intended to improve oral bioavailability $[59,60]$. These may include effect of salt formation, particle size reduction, amorphous and lipid formulations. In addition, in silico methods may be used to aid initial selection of suitable excipients, such as polymer and surfactants for drug solubilization. The latter includes molecular dynamics simulations capable of generating solubility and Flory-Huggins interaction parameters values as a means of predicting drug miscibility in drug-polymer binary mixtures. For solute-solvent pairs, it has been reported that values for difference in solubility parameters of less than $7.5\left(\mathrm{~J} / \mathrm{cm}^{3}\right)^{1 / 2}$ indicate good solubility of solute in the solvent. Furthermore, thermodynamic predictions can provide energetically favorable ranges of API/ polymer drug loading levels that result in either stable solid solution (up to about 10\% drug loading) or metastable supersaturated solid solutions (up to approximately 40\% DL) [61, 62].

Formulation design requires understanding of the API phase properties and its solubility/ miscibility in excipients coupled with the 
application of appropriate pharmaceutical processing technologies designed to enhance apparent solubility (i.e., maintain a supersaturated state) and stabilize API solid-state transitions.

In-vitro dissolution of weak bases and their salts in simulated gastric and intestine fluid media (FaSSIF and FeSSIF containing bile salts) [63] is particularly relevant to indicate how dissolution of drug dose in luminal fluid volume is effected by solubility and supersaturation/ precipitation behavior of the free base in the GI pH range covering pre/postprandial conditions [64, 65]. For example, initial incomplete dissolution of very low soluble weak bases (and their salts) in acidic environment of fasting stomach may lead to rapid supersaturation and precipitation of the free base not likely to be dissolved/absorbed in the upper small intestine. Also, poorly soluble weak bases typically show higher rate and extent of in-vitro dissolution in FeSSIF media (as compared to FaSSIF media) indicating the solubilizing effect of bile salts/lecithin present in such media and hence forecast presence of significant food effect on their oral bioavailability profile. On the other hand, sparingly soluble weakly acidic molecules that have high permeability are well absorbed at the intestinal absorption site provided its relevant pKa (carboxylic acids 4-6) lies within a range to permit ionization of the unionized molecule [66]. Several broad categories of excipient that are commonly utilized in various solubilization technologies, such as nanomilling, liquid formulations, and amorphous solid dispersion technologies are given in Table 5 [36, 55, 67-78].

The chemical compatibility of the APIs with each other and with excipients may not be easily predicted from available chemical structure and known degradation pathways. Similarly, consolidation characteristics and flow properties of composite powder and granule blends are not known. Preliminary accelerated stability and compaction simulation design-of-experiment (DOE) studies are flagged for experimental evaluation in the feasibility assessment exercise.

There may be a need to formulate FDC of drugs that are designed to have different diffusion and dissolution kinetics in the same dosage form. Technology approaches used to achieve modified or sustained drug release can vary greatly and may include formulation of multilayer tablets having separate immediate release and modified-release drug layers either as solid granulation layers or as API film layer coated over an uncoated matrix core or over a tablet core with a polymer film coating [51]. Delayed-release, sustained-release and immediate-release multiparticulates alone or in combination have been designed and encapsulated as active containing particulates, or as nonpareil seeds coated with successive API and polymer film layers to impart different drug release characteristics for actives that are either embedded within the particulates or are spray coated as a film.

Pharmaceutical processing technologies needed to manufacture FDC with both immediate and sustained release performance are more complex and the need to conduct well-controlled process characterization studies should be documented in the feasibility assessment plan. Statistical experimental design and implementation of process monitoring and control strategies such as process analytic tools are employed and repeated at different scales to empirically derive the mathematical relationship/s between and amongst critical material and process parameters affecting product quality attributes, and to provide an estimate of process variability. Such DOE studies may eventually lead to determination of the design space of critical material and process 


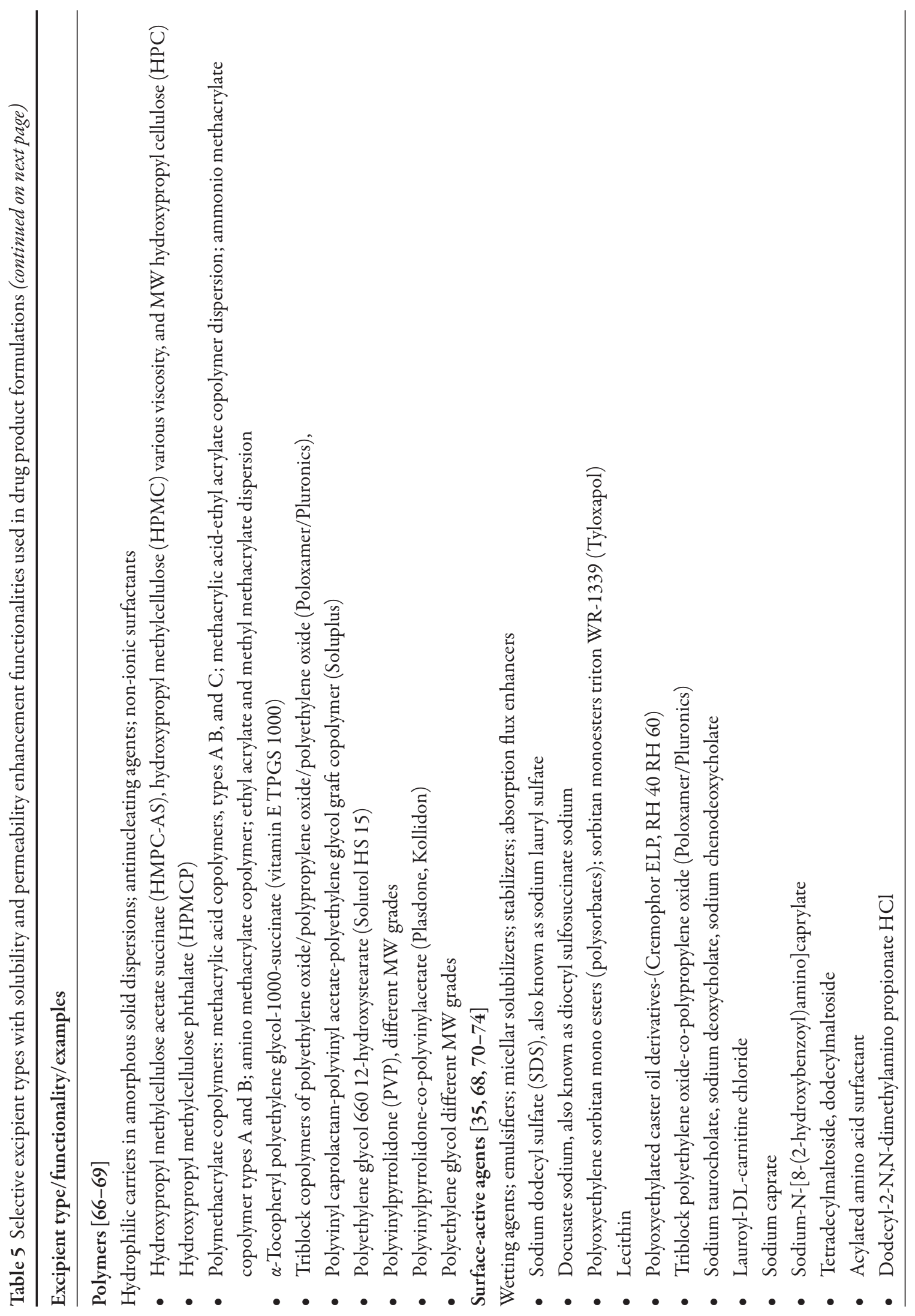




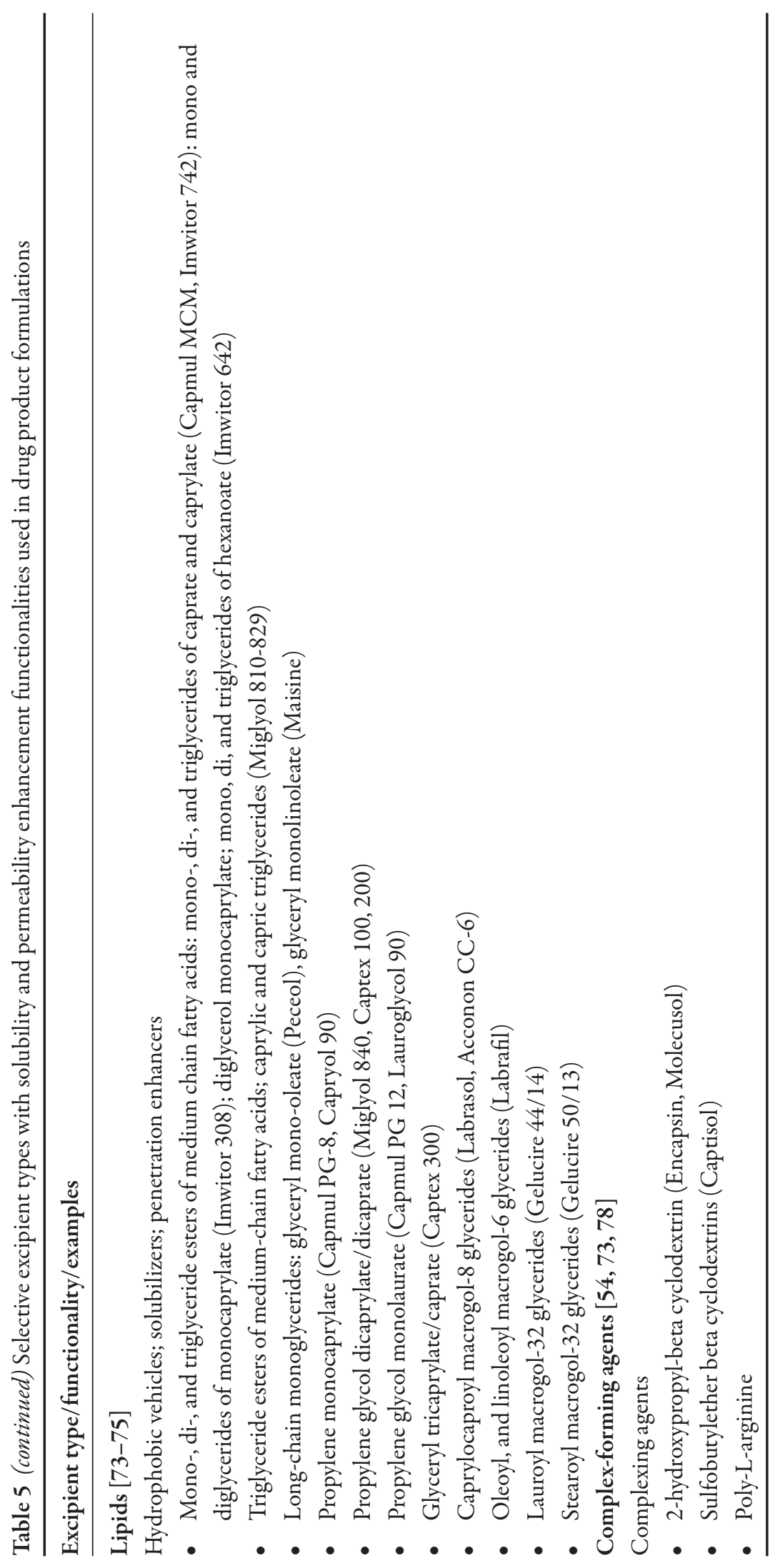


parameters that bounds the controlled operating conditions capable of consistently producing product meeting CQA specifications [52, 79]. In other aspects, a range of FDC drug loading levels (based on type of formulation and technology being employed) and required potencies can be used a priori to estimate overall oral dosage unit size, and hence, its ease of swallowing and patient acceptability limitations of dosing single or multiple units.

\section{IP Assessment}

It is important to analyze the proposed combination therapy against potential competitive therapies and FDC technologies that are in the pipeline, as these competitive technologies may appear well before the proposed FDC reaches the market place. Furthermore, a FDC may combine an innovator's own therapeutic agent with a generically available active component which may still be subject to unexpired formulation patent or to API physical form patent such as a different hydrate, solvate, or polymorph. In these situations, the paper feasibility exercise should include a preliminary understanding of all applicable IP issues and limitations in both API phase and formulation design in order to streamline development effort.

This can be challenging particularly if generic API of interest has low solubility or has to be formulated as sustained release in the FDC. Such methods of composition and process are usually subject to rich IP protection and patent extensions. These factors are critically reviewed as they may restrict or affect the process route, composition, and technologies that can be employed to design the proposed FDC with its target bioperformance. In particular, a component of regulatory approval relies on establishing bioequivalent performance of the newly formulated FDC with respect to the original reference listed drug (RLD) as defined by FDA in its Orange Book [16].

\section{Preclinical Safety Pharmacology Considerations}

The need for preclinical toxicology data may predicate on having prior co-administration studies, which have demonstrated efficacy and safety of the proposed combination therapy for the target indication in clinical studies of patient populations. Regulatory requirements for conducting preclinical safety pharmacology studies and drug-drug pharmacokinetic interaction studies must be considered for novel combinations even if the individual components are known (for which no concomitant use data exists) and for those involving a new investigational drug.

Before initiating first-in-man clinical investigation of the combination product, it is necessary that the safety profiles of individual APIs are established. The latter includes conventional pharmacology and toxicology studies, such as genotoxicity, mutagenicity, and immunotoxicity. These considerations include establishing maximum-tolerated doses (or in some cases maximum feasible dose) of the drugs alone as well as their combination in GLP (good laboratory practices) acute and repeat dose toxicity studies in preclinical animal species. Maximum-tolerated single dose establishes preliminary safety margin related to proposed human dose. The no observed adverse effect level (NOAEL) demonstrates adequacy of safety margin based on projected exposures in humans at proposed therapeutic range and above milligram per kilogram doses of each active constituent $[6,7]$.

Clinical Development and Regulatory Strategy Although several scenarios are possible depending on the type of FDC being developed, 
a pilot bioavailability study in man is conducted early on in development to evaluate the bioperformance of the combination drugs being formulated into a single unit. The pharmacokinetic bioavailability study is designed as a crossover, open-label, single-dose study in a small number of healthy subjects to determine the comparative bioavailability of the FDC dosage form versus that of the individual active entities co-administered, on separate occasions, at corresponding doses. Subjects are randomly assigned to each of possible sequences with adequate washout period between product administrations. Furthermore, since the purpose of a probe pharmacokinetic study is to assess the probability of success of an eventual definitive bioequivalence study, wider comparative bioavailability (74-143\%) instead of bioequivalent (80-125\%) acceptance limits may be used. These limits bound the 90\% confidence interval of the geometric least square mean ratios (GMR) of both area under curve $\left(\mathrm{AUC}_{0-\infty}\right)(\mu \mathrm{M} . \mathrm{hr})$, and $\mathrm{C}_{\max }(\mathrm{nM})$ of FDC over that of each of its corresponding active components. Statistics pertaining to $\mathrm{C}_{24^{\circ} \mathrm{hr}}(\mathrm{nM})$, $\mathrm{T}_{\max }$ (hour) and apparent $\mathrm{t}_{1 / 2}$ (hour) may be included to inform on the shape of the systemic exposure profiles.

The probe pharmacokinetic study could be designed to include a fed arm in the same study subjects to determine the effect of nutrients on drug absorption and pharmacokinetic endpoints. The fed arm of the study is usually carried out by administering a standard meal (breakfast or evening meal) just prior to dosing. If more than one dose level is being investigated the fed arm may test the worst-case scenario by determining the effect of food/nutrients on pharmacokinetic parameters at only the highest dose. It is noted that this type of fed versus fasted pharmacokinetic study does not constitute a food effect study. A separate food effect study of the FDC may be warranted where the effects of high-fat meal versus low-fat meal on systematic exposure and pharmacokinetic endpoints are evaluated.

For combination products of previously approved drugs, ultimately a sufficiently powered definitive bioequivalence study is required as part of the regulatory filing strategy [80-82]. Drug products are considered therapeutically equivalent if they meet FDA regulatory criteria of pharmaceutical equivalence and bioequivalence. The definitive bioequivalence study uses the optimized to-bemarketed formulation of FDC (usually at highest strength) versus co-administration of the appropriate reference drug products such as innovator's own approved drugs or in the case of generics, the reference listed drugs at the strength specified in the Orange Book [16]. Chen et al. [83] proposed that therapeutic equivalence may be established by matching the in vivo drug delivery profile between the reference and the comparator drug products by first identifying critical variables that serve as in vitro markers for characterizing the desired drug delivery profile in vivo.

As an example, an accelerated regulatory filing strategy may consist of a complete phase 1 pharmacokinetic program, pivotal bioequivalent studies plus one or more clinical noninferiority efficacy studies (particularly for EU), wherein bridging to clinical safety and tolerability data in the original new drug application (NDA) submissions are possible. Haider et al. [84] have proposed a new replicate study design for adjusting the standard bioequivalence limits for drug products for which the coefficient of within subject variability is greater than $30 \%$. This has the advantage of alleviating the need for large number of subjects normally required for traditional bioequivalence trials. 
When the FDC product contains one or more API formulated to possess a modified release profile a new NDA submission may generally be required, if this represents the first modified release formulation of the same previously approved immediate-release drug product. However, for any subsequent FDC in which the modified release formulation of an active component is considered to be pharmaceutically equivalent to the marketed controlled-release product (at the specified strength) aforementioned bioavailability and bioequivalent requirements may apply. Furthermore, the bioavailability studies are designed to establish: 1) absence of occurrence of any dose dumping, and 2) consistent pharmacokinetic performance between individual dosage units after both a fasted and fed single-dose study.

For an immediate-release/modified-release FDC (such as a bilayer tablet), an additional complicating factor may arise if no prior clinical data are available for the co-administration of the individual actives formulated as separate immediate release and modified release drug product entities. However, in one scenario, assuming that prior co-administration data does exist for immediate release versions of the actives, a possible resolution could be to provide supportive scientific literature that show non-inferior efficacy and similar safety of known immediate release and modified release product versions of the same API.

\section{CONCLUSION}

FDCs continue to be in the forefront of innovations and enhanced drug therapies for the treatment and management of complex diseases such as diabetes, hepatitis C, HIV/ AIDS, and hypertension. The rationale for the development of FDCs may include treatment of two closely related diseases, patients insufficiently controlled by optimally dosed individual mono-therapies, and substitution of FDC versus free combination. Other considerations in the development of best-inclass commercial combination products include treatment paradigms in therapeutic area, patient compliance and impact of personalized medicine, shifts in market and cost drivers, preclinical and pharmaceutical development and manufacturing, competitive IP landscape, and clinical development and regulatory filing strategy. In the future, selection and screening of new drug combinations may be based on predictions from both mechanisms and targets of drug action as well as pharmacokinetic ADME properties. Combination drug therapies with individualized optimization are likely to become a major focus.

\section{ACKNOWLEDGMENTS}

N.P. is the guarantor for this article, and takes responsibility for the integrity of the work as a whole. The author is currently employed at Novo Nordisk A/S and is solely responsible for the contents of the article. No other person met ICMJE authorship criteria for this manuscript. Editorial assistance in the preparation of this manuscript was provided at no cost by Bradford Challis.

Conflict of interest. The author has no commercial or other associations that might pose a conflict of interest in connection with this work, and there were no funding sources supporting the work.

Open Access. This article is distributed under the terms of the Creative Commons Attribution Noncommercial License which permits any noncommercial use, distribution, and reproduction in any medium, provided the original author(s) and source are credited. 


\section{REFERENCES}

1. Jia J, Zhu F, Ma X, et al. Mechanisms of drug combinations: interaction and network perspectives. Nat Rev Drug Discov. 2009;8:111-28.

2. Keith CT, Borisy AA, Stockwell BR. Multicomponent therapeutics for networked systems. Nat Rev Drug Discov. 2005;4:71-8.

3. Lauritsen KJ, Nguyen T. Combination products regulation at the FDA. Clin Pharmacol Ther. 2009;85:468-70.

4. O'Neill WW, Leon MB. Drug-eluting stents: costs versus clinical benefit. Circulation. 2003;107:300811.

5. Shmulewitz A, Langer R. The ascendance of combination products. Nat Biotechnol. 2006;24:277-80.

6. EMEA Committee for Medicinal Products for Human Use (CHMP). Guideline on the nonclinical development of fixed combinations of medicinal products; January 24, 2008. Available at: http://www.ema.europa.eu/docs/en_GB/ document_library/Scientific_guideline/2009/10/ WC500003976.pdf. Accessed Nov 152011.

7. Guidance for Industry and FDA Staff: Early Development Considerations for Innovative Combination Products; September 2006. Available at: http://www.fda.gov/downloads/ RegulatoryInformation/Guidances/ucm 126054. pdf. Accessed Nov 152011.

8. Chrysant SG. Triple-drug, fixed-dose combinations for the treatment of hypertension: focus on olmesartan/amlodipine/hydrochlorothiazide combination. Drugs Today (Barc). 2011;47:197-206.

9. Bennett WL, Maruthur NM, Singh S, et al. Comparative effectiveness and safety of medications for type 2 diabetes: an update including new drugs and 2-drug combinations. Ann Intern Med. 2011;154:602-13.

10. Florentin M, Elisaf MS, Mikhailidis DP, Liberopoulos EN. Drug combinations for dyslipidemia and obesity treatment in metabolic syndrome. Curr Pharm Des. 2009;15:3446-62.

11. Lotsch J, Geisslinger G. Low-dose drug combinations along molecular pathways could maximize therapeutic effectiveness while minimizing collateral adverse effects. Drug Discov Today. 2011;16:1001-6.
12. Scourfield A, Waters L, Nelson M. Drug combinations for HIV: what's new? Expert Rev Anti Infect Ther. 2011;9:1001-11.

13. Connor J, Rafter N, Rodgers A. Do fixed-dose combination pills or unit-of-use packaging improve adherence? A systematic review. Bull World Health Organ. 2004;82:935-9.

14. Maggiolo F, Ripamonti D, Arici C, et al. Simpler regimens may enhance adherence to antiretrovirals in HIV-infected patients. HIV Clin Trials. 2002;3:371-8.

15. Drug interactions are risky for older adults. Seven common drug combinations could cause serious side effects, a new study shows. Duke Med Health News. 2009;15:4-5. PMID:19639660.

16. Electronic Orange Book: Approved drug products with therapeutic equivalence evaluations. U.S. Department of Health and Human Services, Food and Drug Administration, Center for Drug Evaluations and Research, Office of Pharmaceutical Sciences, Office of Generic Drugs. Available at: http://www.fda.gov/Drugs/InformationOnDrugs/ ucm129662.htm. Accessed Nov 252011.

17. Jackson G. The Polypill - multiple drug combinations are not the answer. Int J Clin Pract. 2011;65:1113-4.

18. Bailey CJ, Day C. Fixed-dose single tablet antidiabetic combinations. Diabetes Obes Metab. 2009;11:527-33.

19. Bangsberg DR, Kroetz DL, Deeks SG. Adherenceresistance relationships to combination HIV antiretroviral therapy. Curr HIV/AIDS Rep. 2007;4:65-72.

20. Sasich LD, Wolfe SM, Pearson C, et al. The National Council on Patient Information and Education. JAMA. 1997;278:1491-2.

21. Bangalore S, Shahane A, Parkar S, Messerli FH. Compliance and fixed-dose combination therapy. Curr Hypertens Rep. 2007;9:184-9.

22. Bergmann JF. Review: fixed-dose drug combinations improve medication compliance compared with free-drug regimens. Evid Based Med. 2008;13:18.

23. Bangalore S, Kamalakkannan G, Parkar S, Messerli FH. Fixed-dose combinations improve medication compliance: a meta-analysis. Am J Med. 2007;120:713-9. 
24. Sadee W, Dai Z. Pharmacogenetics/genomics and personalized medicine. Hum Mol Genet. 2005;14 Spec No. 2:R207-14.

25. Woodcock J. The prospects for "personalized medicine" in drug development and drug therapy. Clin Pharmacol Ther. 2007;81:164-9.

26. Sjoqvist F, Eliasson E. The convergence of conventional therapeutic drug monitoring and pharmacogenetic testing in personalized medicine: focus on antidepressants. Clin Pharmacol Ther. 2007;81:899-902.

27. Draft Guidance for Industry and Food and Drug Administration Staff: In Vitro Companion Diagnostic Devices, July 2011. Available at: http://www.fda.gov/downloads/ MedicalDevices/DeviceRegulationandGuidance/ GuidanceDocuments/UCM262327.pdf. Accessed Feb 232012.

28. Schmidt C. Larger companies dominate cancer companion diagnostic approvals. Nat Biotechnol. 2011;29:955-7.

29. Gurwitz D, Lunshof JE, Altman RB. A call for the creation of personalized medicine databases. Nat Rev Drug Discov. 2006;5:23-6.

30. Sadee W. Drug therapy and personalized health care: pharmacogenomics in perspective. Pharm Res. 2008;25:2713-9.

31. van der Greef J, Hankemeier T, McBurney RN. Metabolomics-based systems biology and personalized medicine: moving towards $n=1$ clinical trials? Pharmacogenomics. 2006;7:108794.

32. Sarachan BD, Simmons MK, Subramanian P, Temkin JM. Combining medical informatics and bioinformatics toward tools for personalized medicine. Methods Inf Med. 2003;42:111-5.

33. Wilke RA, Berg RL, Peissig P, et al. Use of an electronic medical record for the identification of research subjects with diabetes mellitus. Clin Med Res. 2007;5:1-7.

34. FDA Table of Pharmacogenomic Biomarkers in Drug Label; Nov 23, 2011. Available at: http://www.fda.gov/Drugs/ScienceResearch/ ResearchAreas/Pharmacogenetics/ucm083378.htm. Accessed Nov 252011.

35. Richman D, Wrin MT, Little S, et al., inventors; Monogram Biosciences, Inc., assignee. Method of evaluating a patient antibody response to envelope proteins of a virus. United States patent 7247439 . Jul 24, 2007.

36. Strickley RG, Oliyai R. Solubilizing Vehicles for Oral Formulation Development. Springer New York; 2007;257-308.

37. Garber K. Genomic medicine. Gene expression tests foretell breast cancer's future. Science. 2004;303:1754-5.

38. Kaklamani V. A genetic signature can predict prognosis and response to therapy in breast cancer: Oncotype DX. Expert Rev Mol Diagn. 2006;6:8039.

39. Sotiriou C, Piccart MJ. Taking gene-expression profiling to the clinic: when will molecular signatures become relevant to patient care? Nat Rev Cancer. 2007;7:545-53.

40. Sparano JA, Paik S. Development of the 21-gene assay and its application in clinical practice and clinical trials. J Clin Oncol. 2008;26:721-8.

41. West M, Ginsburg GS, Huang AT, Nevins JR. Embracing the complexity of genomic data for personalized medicine. Genome Res. 2006;16:559_ 66.

42. Krynetskiy E, McDonnell P. Building individualized medicine: prevention of adverse reactions to warfarin therapy. J Pharmacol Exp Ther. 2007;322:427-34.

43. Hegele RA, Guy J, Ban MR, Wang J. NPC1L1 haplotype is associated with inter-individual variation in plasma low-density lipoprotein response to ezetimibe. Lipids Health Dis. 2005;4:16.

44. Simon JS, Karnoub MC, Devlin DJ, et al. Sequence variation in NPC1L1 and association with improved LDL-cholesterol lowering in response to ezetimibe treatment. Genomics. 2005;86:648-56.

45. Reitman ML, Schadt EE. Pharmacogenetics of metformin response: a step in the path toward personalized medicine. J Clin Invest. 2007;117:1226-9.

46. Ross JS, Fletcher JA. The HER-2/neu oncogene in breast cancer: prognostic factor, predictive factor, and target for therapy. Oncologist. 1998;3:237-52.

47. Abrahams E, Ginsburg GS, Silver M. The Personalized Medicine Coalition: goals and strategies. Am J Pharmacogenomics. 2005;5:34555. 
48. Ginsburg G, Angrist M. The future may be closer than you think: a response from the PMC to the Royal Society's report on personalized medicine. Personalized Medicine. 2006;3:119-23.

49. Newman J, Grobman WA, Greenland P. Combination polypharmacy for cardiovascular disease prevention in men: a decision analysis and cost-effectiveness model. Prev Cardiol. 2008;11:3641.

50. Dahl TC, Menning MM, Oliyai R, Yang T, inventors; Gilead Sciences, Inc., assignee. Method and composition for pharmaceutical product United States patent 0077295. Apr 5, 2007.

51. Pourkavoos N, inventor; Pharmaceutical compositions of a combination of metformin and a dipeptidyl peptidase-IV inhibitor. United States patent 2010/0330177. Dec 30, 2010.

52. Yu LX. Pharmaceutical quality by design: product and process development, understanding, and control. Pharm Res. 2008;25:781-91.

53. Martinez MN, Amidon GL. A mechanistic approach to understanding the factors affecting drug absorption: a review of fundamentals. J Clin Pharmacol. 2002;42:620-43.

54. Alsenz J, Kansy M. High throughput solubility measurement in drug discovery and development. Adv Drug Deliv Rev. 2007;59:546-67.

55. Avdeef A, Bendels S, Tsinman O, Tsinman K, Kansy M. Solubility-excipient classification gradient maps. Pharm Res. 2007;24:530-45.

56. Chen H, Zhang Z, McNulty C, et al. A highthroughput combinatorial approach for the discovery of a cremophor EL-free paclitaxel formulation. Pharm Res. 2003;20:1302-8.

57. Kuentz M. Drug absorption modeling as a tool to define the strategy in clinical formulation development. AAPS J. 2008;10:473-9.

58. Thelen K, Coboeken K, Willmann S, et al. Evolution of a detailed physiological model to simulate the gastrointestinal transit and absorption process in humans, Part 1: Oral solutions. J Pharm Sci. 2011;100:5324-45.

59. Kesisoglou F, Wu Y. Understanding the effect of API properties on bioavailability through absorption modeling. AAPS J. 2008;10:516-25.

60. Okumu A, DiMaso M, Lobenberg R. Dynamic dissolution testing to establish in vitro/in vivo correlations for montelukast sodium, a poorly soluble drug. Pharm Res. 2008;25:2778-85.
61. Faller B, Ertl P. Computational approaches to determine drug solubility. Adv Drug Deliv Rev. 2007;59:533-45.

62. Huynh L, Grant J, Leroux JC, Delmas P, Allen C. Predicting the solubility of the anti-cancer agent docetaxel in small molecule excipients using computational methods. Pharm Res. 2008;25:14757.

63. Bakatselou V, Oppenheim RC, Dressman JB. Solubilization and wetting effects of bile salts on the dissolution of steroids. Pharm Res. 1991;8:1461-9.

64. Dressman JB, Vertzoni M, Goumas K, Reppas C. Estimating drug solubility in the gastrointestinal tract. Adv Drug Deliv Rev. 2007;59:591-602.

65. Kostewicz ES, Brauns U, Becker R, Dressman JB. Forecasting the oral absorption behavior of poorly soluble weak bases using solubility and dissolution studies in biorelevant media. Pharm Res. 2002;19:345-9.

66. Kalantzi L, Goumas K, Kalioras V, et al. Characterization of the human upper gastrointestinal contents under conditions simulating bioavailability/bioequivalence studies. Pharm Res. 2006;23:165-76.

67. Constantinides PP, Han J, Davis SS. Advances in the use of tocols as drug delivery vehicles. Pharm Res. 2006;23:243-55.

68. Leuner C, Dressman J. Improving drug solubility for oral delivery using solid dispersions. Eur J Pharm Biopharm. 2000;50:47-60.

69. Li P, Zhao L. Developing early formulations: practice and perspective. Int J Pharm. 2007;341:119.

70. Curatolo W, Nightingale JA, Herbig SM. Utility of hydroxypropylmethylcellulose acetate succinate (HPMCAS) for initiation and maintenance of drug supersaturation in the GI milieu. Pharm Res. 2009;26:1419-31.

71. Buyuktimkin S, Buyuktimkin N, inventors; NexMed Holdings, Inc., assignee. Crystalline salts of dodecyl 2-(N, N-dimethylamino)-propionate. United States patent 6,118,020. Sep 12, 2000.

72. Maher S, Kennelly R, Bzik VA, et al. Evaluation of intestinal absorption enhancement and local mucosal toxicity of two promoters. I. Studies in isolated rat and human colonic mucosae. Eur J Pharm Sci. 2009;38:291-300. 
73. Steinert RE, Poller B, Castelli MC, et al. Orally administered glucagon-like peptide-1 affects glucose homeostasis following an oral glucose tolerance test in healthy male subjects. Clin Pharmacol Ther. 2009;86:644-50.

74. Strickley RG. Solubilizing excipients in oral and injectable formulations. Pharm Res. 2004;21:20130 .

75. Whitehead K, Karr N, Mitragotri S. Safe and effective permeation enhancers for oral drug delivery. Pharm Res. 2008;25:1782-8.

76. Gao P, Morozowich W. Development of supersaturatable self-emulsifying drug delivery system formulations for improving the oral absorption of poorly soluble drugs. Expert Opin Drug Deliv. 2006;3:97-110.

77. Hauss DJ. Oral lipid-based formulations. Adv Drug Deliv Rev. 2007;59:667-76.

78. Brewster ME, Loftsson T. Cyclodextrins as pharmaceutical solubilizers. Adv Drug Deliv Rev. 2007;59:645-66.

79. Guidance for Industry and FDA: Current good manufacturing practice for combination products, September 2004. Available at:
http://www.fda.gov/downloads/Drugs/

GuidanceComplianceRegulatoryInformation/

Guidances/ucm070268.pdf. Accessed Nov 252011.

80. EMEA Committee for Proprietary Medicinal Products (CHMP). Guideline on the Investigation of Bioequivalence; July 24, 2008. Available at: http://www.emea.europa.eu/docs/en_GB/ document_library/Scientific_guideline/2009/09/

WC500003011.pdf. Accessed Nov 152011.

81. FDA Center for Drug Evaluation and Research. Guidance for industry: bioavailability and bioequivalence studies for orally administered drug products - general considerations; March 2003. Available at: http://www.fda.gov/downloads/Drugs/ GuidanceComplianceRegulatoryInformation/ Guidances/ucm070124.pdf. Accessed Nov 152011.

82. Chen ML, Shah V, Patnaik R, et al. Bioavailability and bioequivalence: an FDA regulatory overview. Pharm Res. 2001;18:1645-50.

83. Chen ML, Lee VH. Equivalence-by-design: targeting in vivo drug delivery profile. Pharm Res. $2008 ; 25: 2723-30$.

84. Haidar SH, Davit B, Chen ML, et al. Bioequivalence approaches for highly variable drugs and drug products. Pharm Res. 2008;25:237-41. 